OPEN ACCESS

Edited by:

Nihal Ahmad,

University of Wisconsin-Madison,

United States

Reviewed by:

loana Cosgarea,

Newcastle University, United Kingdom

Gagan Chhabra,

University of Wisconsin-Madison,

United States

*Correspondence:

Guan Jiang

dr.guanjiang@xzhmu.edu.cn

Specialty section:

This article was submitted to

Skin Cancer,

a section of the journal

Frontiers in Oncology

Received: 02 October 2020

Accepted: 07 December 2020

Published: 26 January 2021

Citation:

Chen $X$, Hou Y, Chen $C$ and Jiang G (2021) Basal Cell Carcinoma

of the External Genitalia:

A Population-Based Analysis.

Front. Oncol. 10:613533.

doi: 10.3389/fonc.2020.613533

\section{Basal Cell Carcinoma of the External Genitalia: A Population-Based Analysis}

\author{
Xi Chen ${ }^{1}$, Yulong Hou ${ }^{1}$, Can Chen ${ }^{2}$ and Guan Jiang ${ }^{1 *}$ \\ ${ }^{1}$ Department of Dermatology, Affiliated Hospital of Xuzhou Medical University, Xuzhou, China, ${ }^{2}$ Hebei Medical University, \\ Shijiazhuang, China
}

Introduction: Basal cell carcinoma (BCC) located on the genitalia is rare; data on the clinicopathologic features and survival outcomes are only available through case reports and small case series studies.

Purpose: This study aimed to explore the epidemiology and identify the prognostic factors of genital BCCs.

Methods: We queried the 18 registries of the Surveillance, Epidemiology, and End Results database for patients with primary BCCs of the genital skin from 2000 through 2017. The primary endpoint was overall survival (OS) and disease specific survival (DSS). Kaplan-Meier survival analysis was conducted to assess the impact of clinicopathological variables on OS and DSS. Multivariate Cox proportional hazards model was performed to evaluate risk factors for OS.

Results: A total of 1,607 cases of genital BCCs were identified. The cohort was composed of 1,352 women (84.1\%) and 255 men (15.9\%). The median (P25, P75) age of the entire cohort was 73(63-82)years. White patients accounted for $87.2 \%$ of the cases. For women and men, the most common site of involvement was the labia majora $(89.6 \%)$ and scrotum (74.5\%), respectively. The majority of patients with genital BCC had localized disease (75.5\%). Kaplan-Meier survival analysis showed that female genital BCCs experienced better DSS than men (209.1 months vs 194.8 months); for men, BCCs located on the scrotum had better DSS and OS than those on the penis $(P<0.05$ for both endpoints). All patients with distant disease died of disease-specific death, and the average survival time was 8.2 months. Multivariate analysis revealed that age, primary site, and stage were independent determinants of OS for men, while tumor size, histologic subtype, and race were not. For women, factors associated with worse OS included increasing age, tumor size more than $2 \mathrm{~cm}$, and distant disease; factors associated with a decreased risk included "other" and "unknown" races.

Conclusion: The prognosis of genital BCCs is excellent, while the survival of distant disease is very poor. Despite similar clinicopathologic features and overall survival outcomes, men and women should be treated as two different entities when making survival predictions. 


\section{INTRODUCTION}

Basal cell carcinoma (BCC) is the most common malignancy and accounts for $75 \%$ non-melanoma skin cancers $(1,2)$. Ultraviolet (UV) radiation is considered the prime factor in its pathogenesis; unsurprisingly, more than $80 \%$ of BCCs are located on the sunexposed skin of the elderly, especially in the head and neck regions $(3,4)$. BCCs can also involve areas with less sun exposure such as the trunk (3). However, only rare BCCs occur on sunprotected sites, and genital BCCs account for less than $1 \%$ of all BCCs, according to published literature $(2,4)$.

Given the extremely rare occurrence of genital BCCs, most of the records on genital BCCs are single cases or small case series, which most likely represent the extreme situation in clinical practice and lack of universality. To our knowledge, no comprehensive study on genital BCCs has performed a direct comparison between women and men, as well as between genital subsites via Kaplan-Meier analysis and multivariate analysis. Herein, detailed analyses were conducted utilizing the data from the Surveillance, Epidemiology, and End Results (SEER) program database. This analysis will provide important counseling value in clinical practice.

\section{METHODS}

\section{Patient Selection}

We queried the SEER database's 18 registries for patients diagnosed with primary BCCs of the external genitalia between the years 2000 and 2017. The SEER database contains epidemiologic information on cancer survival and is updated annually by the National Cancer Institute, covering $28 \%$ of the US population. The right to access these data through SEER Stat software was granted by the National Cancer Institute after submitting the signed SEER data-use agreement. Institutional review board approval was not necessary for this study due to publicly accessible data. Patients with genital BCC were identified using the histologic codes 8090/3 (basal cell carcinoma and not otherwise specified [NOS]), 8091/3 (multifocal superficial basal cell carcinoma), 8092/3 (infiltrating basal cell carcinoma, NOS), 8093/3 (basal cell carcinoma, fibroepithelial), and 8097/3 (basal cell carcinoma, nodular), as well as the primary site codes C51.0 (labium majus), C51.1 (labium minus), C51.2 (clitoris), C51.8 (overlapping lesion of vulva), C51.9 (vulva, NOS), C60.0 (prepuce), C60.2 (body of penis), C60.8 (overlapping lesion of penis), C60.9 (penis, NOS), and C63.2 (scrotum, NOS). Patients with BCC but without histologic confirmation were excluded.

\section{Variables}

The following variables were extracted for analysis: sex, age, race, year of diagnosis, primary site, tumor size, histologic type, stage, cause of death, survival months, and vital status. According to the SEER Historic Stage A, stage was coded as localized (confined to the boundary of the primary organ), regional (direct extension to adjacent organs or structures or with regional lymph node involvement), and distant (spread to distant parts of the primary tumor). Race was grouped into the categories of white, black, and others (American Indian/Alaska Native and Asian/Pacific Islander). Tumor size was determined by comprehensive analyses of "CS tumor size (2004-2015)" and "Tumor Size Summary $(2016+)$ ". For the sake of this analysis, the continuous variable age was categorized as follows (years): $\leq 60$, $61-70,71-80$, or $>80$, and tumor size was categorized into two groups $(\mathrm{cm}): \leq 2$ or $>2$. For convenience, body of penis, overlapping lesion of penis, prepuce, and penis NOS were reclassified as penis. Men were divided into the penis group and the scrotum group by anatomic site. Women were divided into the following subgroups based on the location records of the SEER database: labium majus, labium minus, clitoris, overlapping lesion of vulva, and vulva NOS. Histopathological subtypes were classified in accordance with the World Health Organization criteria, including superficial, nodular, infiltrating, fibroepithelial, and BCC NOS.

\section{Statistical Analysis}

Differences in clinicopathologic characteristics between women and men were compared using the Chi-squared tests (categorical variables) and Student's t-test (continuous variables). The primary endpoints were overall survival (OS, defined as the time from diagnosis to death from any cause) and disease specific survival (DSS, defined as the time from diagnosis to a documented death due to BCC). Kaplan-Meier survival analysis was conducted to assess the impact of various variables on OS and DSS (univariate analysis), and the statistical difference in curves was calculated by log-rank tests. Age, gender, and covariates with $\log$-rank $\mathrm{P}<0.2$ were chosen for multivariate analysis. Through Cox proportional hazard models, multivariate analysis was performed to identify important predictors for survival. All statistical analyses were performed using SPSS version 26.0. Two-sided $\mathrm{P}<0.05$ was considered statistically significant.

\section{RESULTS}

\section{Clinicopathologic Characteristics}

A total of 1,607 patients diagnosed with genital primary BCCs from 2000 to 2017 were identified and included in this study. The clinicopathologic characteristics and demographics data of patients are summarized in Tables 1 and 2, respectively. This cohort was composed of 1,352 women $(84.1 \%)$ and 255 men (15.9\%). The female to male ratio was 5.3. Patients over 85 years in the SEER database were simply classified as $85+$. A total of 307 cases over 85 years were included in this study and considered 85 years in the average age estimate for this cohort. The age of women ranged from 20 years to $85+$ years (median 73 years) and man from 34 years to $85+$ years (median 71 years). Of the cohort, $87.2 \%$ were white and $75.5 \%$ had localized disease. The mean tumor size of men was $1.59 \mathrm{~cm}$ (range $0.2-6.0 \mathrm{~cm}$ ), similar to that of women (mean $1.79 \mathrm{~cm}$; range $0.1-9.5 \mathrm{~cm}$ ). Among the patients with known histologic subtypes, most presented with nodular 
TABLE 1 | Clinicopathologic characteristics of patients with genital basal cell carcinoma by sex.

\begin{tabular}{|c|c|c|c|}
\hline Characteristics & Female $(n=1352)$ & Male $(n=255)$ & P-value \\
\hline \multicolumn{4}{|l|}{ Age(years) } \\
\hline Median(P25, P75) & $73(63,82)$ & $71(61,81)$ & 0.059 \\
\hline Range & 20-85+ & $34-85+$ & 0.127 \\
\hline$\leq 60$ & 292 (21.6\%) & 55 (21.6\%) & \\
\hline $61-70$ & 279 (20.6\%) & $69(27.1 \%)$ & \\
\hline $71-80$ & $372(27.5 \%)$ & $64(25.1 \%)$ & \\
\hline$>80$ & 409 (30.3\%) & $67(26.3 \%)$ & \\
\hline Race & & & 0.026 \\
\hline White & $1,187(87.8 \%)$ & 215 (84.3\%) & \\
\hline Black & 40 (2.9\%) & $6(2.4 \%)$ & \\
\hline Others & 79 (5.8\%) & $15(5.9 \%)$ & \\
\hline Unknown & $46(3.4 \%)$ & $19(7.5 \%)$ & \\
\hline \multicolumn{4}{|l|}{ Tumor size(cm) } \\
\hline Mean $\pm \mathrm{SD}$ & $1.8 \pm 0.52$ & $1.6 \pm 1.29$ & 0.166 \\
\hline Range & $0.1-9.5$ & $0.2-6$ & 0.001 \\
\hline$\leq 2$ & 457 (33.8\%) & 67 (26.3\%) & \\
\hline$>2$ & 174 (12.9\%) & 21 (8.2\%) & \\
\hline Unknown & $721(53.3 \%)$ & 167 (65.5\%) & \\
\hline \multicolumn{4}{|l|}{ Primary site } \\
\hline Labium majus & $311(23 \%)$ & - & \\
\hline Labium minus & $18(1.3 \%)$ & - & \\
\hline Clitoris & $8(0.6 \%)$ & - & \\
\hline Overlapping lesion of vulva & $10(0.7 \%)$ & - & \\
\hline Vulva NOS & $1,005(74.3 \%)$ & - & \\
\hline Penis & - & $65(25.5 \%)$ & \\
\hline Scrotum & - & $190(74.5 \%)$ & \\
\hline Histopathology & & & 0.056 \\
\hline Superficial & 24 (1.8\%) & $5(2.0 \%)$ & \\
\hline Nodular & $321(23.7 \%)$ & $69(27.1 \%)$ & \\
\hline Infiltrative & $15(1.1 \%)$ & $4(1.6 \%)$ & \\
\hline Fibroepithelioma & $14(1 \%)$ & $8(3.1 \%)$ & \\
\hline Basal cell carcinoma NOS & 978 (72.3\%) & 169 (66.3\%) & \\
\hline SEER Historic Stage A & & & 0.175 \\
\hline Localized & 1,029 (76.1\%) & $184(72.2 \%)$ & \\
\hline Regional & $30(2.2 \%)$ & $4(1.6 \%)$ & \\
\hline Distant & $3(0.2 \%)$ & $2(0.8 \%)$ & \\
\hline Unknown & $290(21.4 \%)$ & $65(25.5 \%)$ & \\
\hline
\end{tabular}

TABLE 2 | Demographics data of patients.

\begin{tabular}{lcc}
\hline Demographics & $\mathbf{N}$ & $\%$ \\
\hline Total & 1,607 & $100 \%$ \\
Race & 1,402 & \\
White & 46 & $87.2 \%$ \\
Black & 7 & $2.9 \%$ \\
American Indian/Alaska Native & 87 & $0.4 \%$ \\
Asian/Pacific Islander & 65 & $5.4 \%$ \\
Unknown & & $4.0 \%$ \\
Sex & 1,352 & \\
Female & 255 & $84.1 \%$ \\
Male & & $15.9 \%$ \\
Age & 7 & \\
$20-30$ years & 32 & $0.4 \%$ \\
$31-40$ years & 87 & $2.0 \%$ \\
$41-50$ years & 221 & $5.4 \%$ \\
$51-60$ years & 348 & $13.8 \%$ \\
$61-70$ years & 436 & $21.7 \%$ \\
$71-80$ years & 169 & $27.1 \%$ \\
$81-84$ years & 307 & $10.5 \%$ \\
$85+$ years & & $19.1 \%$
\end{tabular}

subtype (84.8\%); others presented with superficial (6.3\%), infiltrative (4.1\%), and fibroepithelioma (4.8\%) subtype. A total of 347 women had specific lesion sites, with the labia majora being the most common involvement (89.6\%). Most male genital BCCs presented in the scrotum (74.5\%).

\section{Survival Analysis by Sex, Age, and Race}

There was no significant difference in OS between women and men, and the overall 5-year survival rates in this study were 79.5 and $77.8 \%$, respectively. Women experienced higher DSS than men (209.1 months $v s 194.8$ months, $\mathrm{P}=0.011)$. Regardless of gender, OS decreased as age increased $(\mathrm{P}<0.0001)$, which is not a surprising finding considering the older age composition of this study. Women with age over 80 years had significantly worse DSS than other age groups $(P<0.0001)$, while there was less prognostic value in age for male DSS $(\mathrm{P}=0.229)$ (Figure 1). For women, whites had the worst OS among all racial groups $(\mathrm{P}<0.0001)$; for men, there was no statistically significant difference in survival between different races (figures not shown).

\section{Survival Analysis by Stage at Diagnosis and Histologic Subtype}

In this study, 1,252 cases with known SEER stage were available, and only five patients were distant disease, accounting for $0.4 \%$. All patients with distant disease died of genital BCC and experienced the lowest survival time (average 8.2 months). For men, patients diagnosed with localized disease experienced relatively high OS (mean 131.6 months) than patients with regional disease (mean 46.3 months). No significant difference in both OS and DSS was found among women with localized and regional disease (Figure 2). Regarding histologic subtype, there were no significant survival differences (figures not shown).

\section{Survival Analysis by Tumor Size and Anatomic Site}

Survival analysis from Kaplan-Meier curves revealed that patients with tumors larger than $2 \mathrm{~cm}$ had worse OS than those with tumors smaller than $2 \mathrm{~cm}$, regardless of gender, while there was no statistical difference in DSS between the two groups (Figure 2). In terms of anatomic site, BCC located on the scrotum had statistically better survival rates than that on the penis, no matter OS or DSS ( $\mathrm{P}<0.05$ for both endpoints). Contrary to male genital BCC, anatomic site had no prognostic value in female survival (Figure 3).

\section{Multivariate Analysis on OS}

After accounting for other factors including race, primary site, tumor stage, and tumor size, multivariate analysis revealed that age at diagnosis was a statistically significant predictor of OS for both women and men. Increasing age had a significant effect on death, with HRs ranging from 3.25 to 16.58 for men, and 2.38 to 25.9 for women. For women, tumor size more than $2 \mathrm{~cm}$ (hazard ratio [HR] 1.53 [95\% CI 1.11-2.10]) and SEER stage at "distant" (HR 10.24 [95\% CI 3.22-32.59]) were associated with worse OS; race "others" and "unknown" were associated with better OS (HR 0.40 and 0.12 , respectively) (Table 3). For man, anatomic 
A

Overall survival

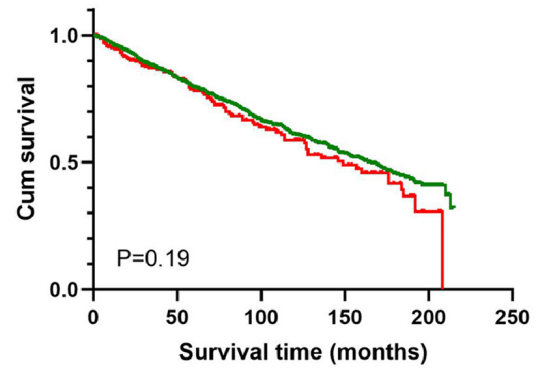

C

Overall survival

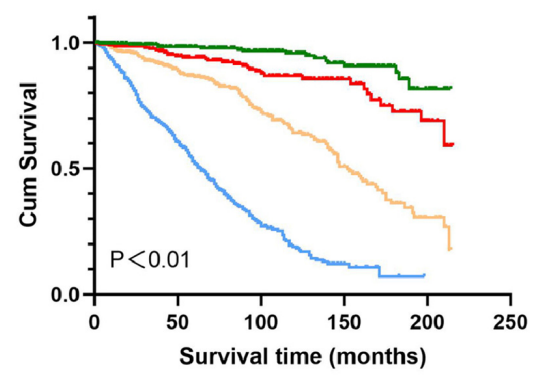

E

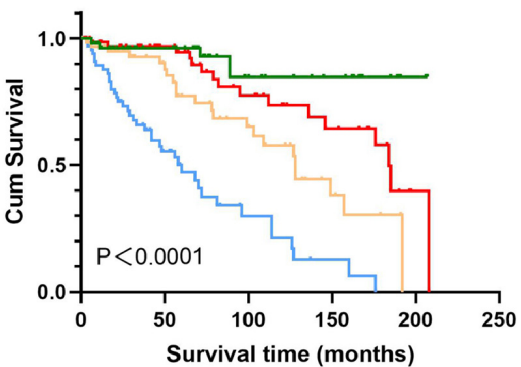

- Female

- Male

- $\leqslant 60 \mathrm{y}$

- 60 70y

$-70 \sim 80 y$

$->80 \mathrm{y}$

$-\leqslant 60 \mathrm{y}$

$-60 \sim 70 y$

$-70 \sim 80 \mathrm{y}$

$->80 \mathrm{y}$
B

Disease specific survival

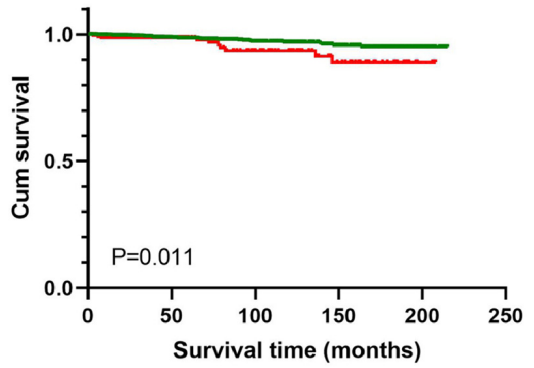

- Female

- Male

D

Disease specific survival

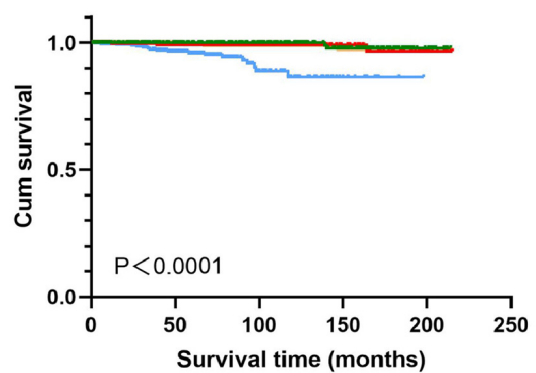

$-\leqslant 60 \mathrm{y}$

$-60 \sim 70 \mathrm{y}$

$-70 \sim 80 \mathrm{y}$

$->80 \mathrm{y}$

$\mathbf{F}$

Disease specific survival

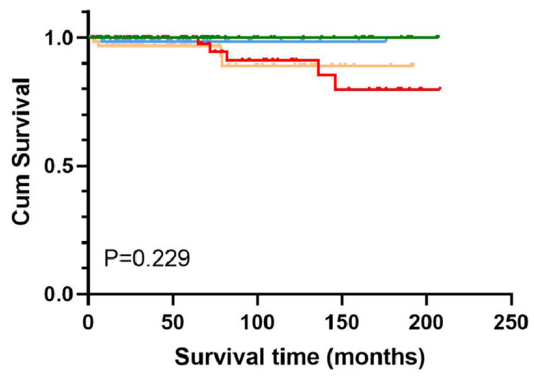

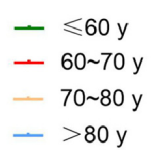

FIGURE 1 | Kaplan-Meier estimates of OS and DSS by sex and age. There was no significant difference in OS between female and male (A), while female experienced higher DSS than men (B). OS decreased as age increased irrespective of sex (C, E). Female over 80 years had significantly worse DSS than other age groups (D); for male, difference in DSS between ages does not reach significance (F). OS, overall survival; DSS, disease-specific survival; BCC, basal cell carcinoma.

subsite and stage were the determinants of survival. Scrotum BCCs had a better prognosis than BCCs located on penis, and the risk of overall death for penis BCCs was almost 1.8 times (HR1.76 [95\% CI 1.06-2.92]) as high as that for scrotum BCCs. Compared to patients with localized disease, patients with regional disease (HR 3.77 [95\% CI 1.12-12.71]) and distant disease experienced significantly higher overall mortality (HR 62.04 [95\% CI 11.1-346.9]) (Table 4). Histologic subtype was not a significant variable for both women and men.

\section{DISCUSSION}

UV-light exposure is the major risk factor in the onset of BCC on sun-exposed areas, and BCC mainly affects Caucasians due to their skin's weak ultraviolet resistance (5). There was almost no record of nude sunbathing in patients with genital BCC in previous retrospective studies or case reports, the relationship of UV with genital BCC is not yet clear.

It is interesting to note that in this study, a majority of patients diagnosed with genital BCC in the US were Caucasians. Of course, it is necessary to further calculate the relative incidence of each race. We assumed that genetic conditions may play a role in the pathogenesis of genital BCC (6). Other risk factors include depressed immune surveillance caused by UV radiation at distant sites or advanced age $(7,8)$, local trauma or burn $(9,10)$, ionizing radiation $(11)$, chronic skin irritation (12), human papillomavirus (HPV) infection (13), and exposure to carcinogens, especially arsenic $(14,15)$. However, only a small percentage of patients in previous case studies were found to have relevant predisposing factors, and HPV was not 
A

Overall survival

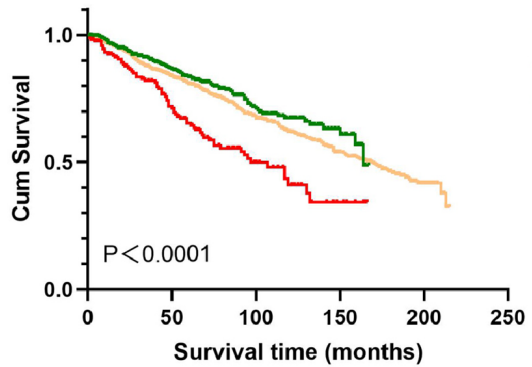

C

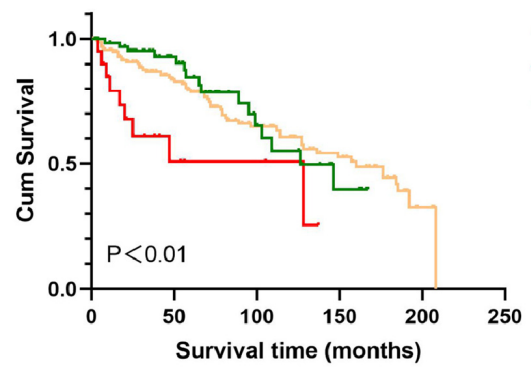

E

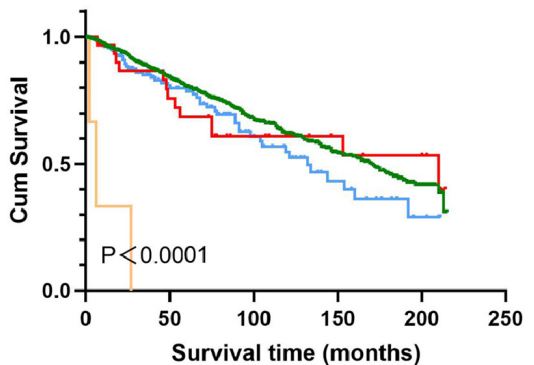

G

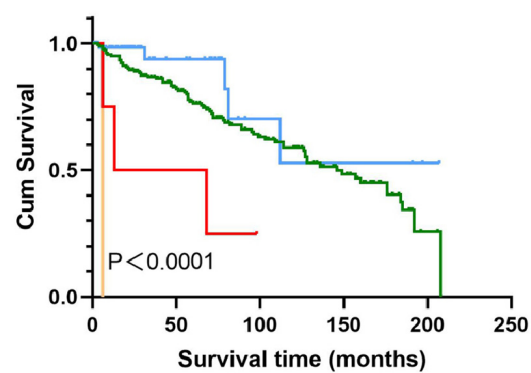

B Disease specific survival

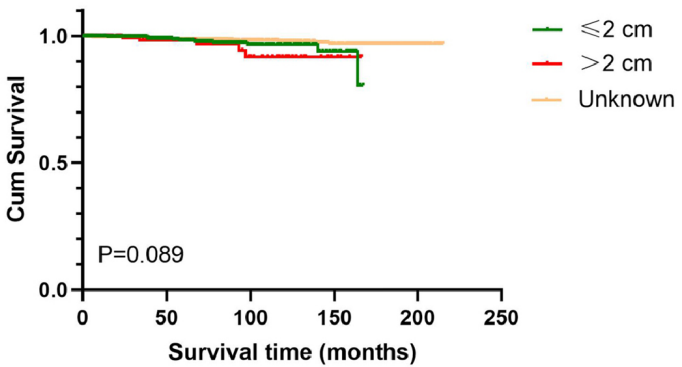

D Disease specific survival

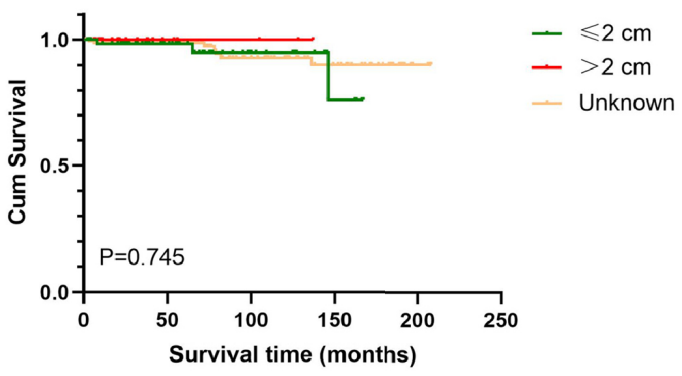

F Disease specific survival

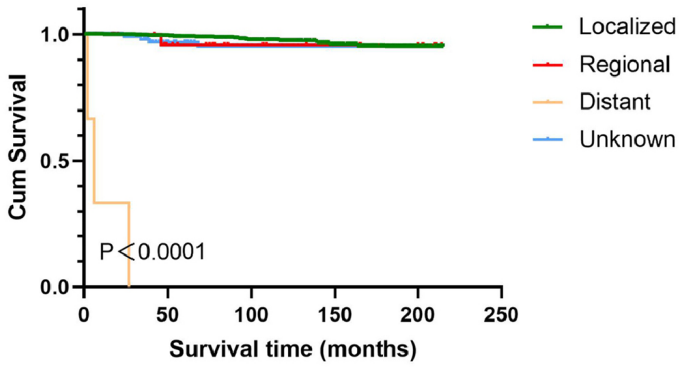

H Disease specific survival

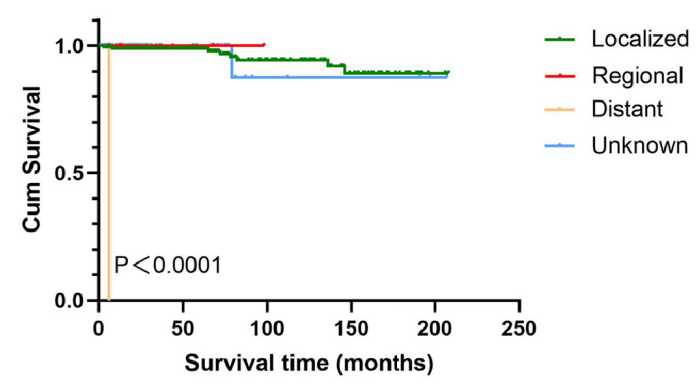

FIGURE 2 | Kaplan-Meier estimates of OS and DSS by tumor size and stage. Patients with tumors $>2 \mathrm{~cm}$ had worse OS than those with tumors $\leq 2 \mathrm{~cm}$ (A, C); tumor size has no prognostic value for DSS in patients with genital BCC (B, D). Patients with distant disease experienced the lowest survival time (E-H). Male with localized disease had relatively high OS than those with regional disease (G), while there was no significant difference in DSS (H). Female with localized and regional disease had similar prognosis (E, F). OS, overall survival; DSS, disease-specific survival; BCC, basal cell carcinoma.

detected in most BCC specimens (12). The etiology of genital BCC remains unknown.

Genital BCCs tend to develop in an older age group, with similar mean age at presentation for women (mean 71 years) and men (mean 69.9 years) in this cohort. Male sex is one risk factor for BCC outside the genitalia, while genital BCCs are predominantly reported in females in previous literature (16, 17). Similarly, in this study, the majority of patients with genital 
A

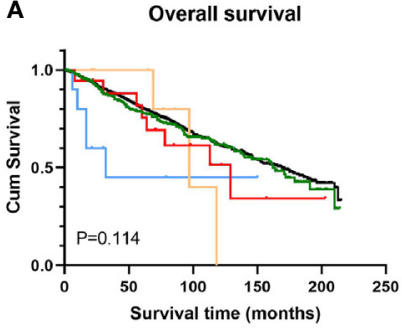

C

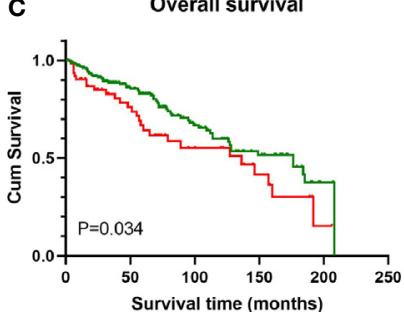

B Disease specific survival

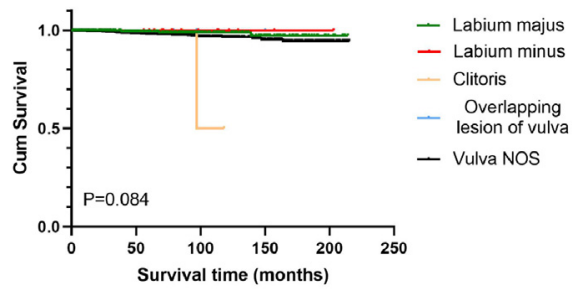

D

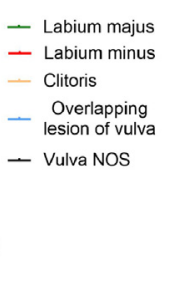

- Scrotum

FIGURE 3 | Survival analysis of genital BCC by subsite. Scrotum BCC had statistically better OS and DSS than penis BCC (C, D); for female genital BCC, anatomic site had little prognostic value (A, B). OS, overall survival; DSS, disease-specific survival; BCC, basal cell carcinoma.

TABLE 3 | Multivariate analyses of overall survival for female genital basal cell carcinoma.

\begin{tabular}{|c|c|c|c|}
\hline Characteristics & HR & $95 \% \mathrm{Cl}$ & P-value \\
\hline \multicolumn{4}{|l|}{ Age } \\
\hline$\leq 60$ & Reference & & \\
\hline $61-70$ & 2.38 & $1.30-4.34$ & 0.005 \\
\hline $71-80$ & 7.08 & $4.20-11.93$ & 0.000 \\
\hline$>80$ & 25.9 & $15.47-43.38$ & 0.000 \\
\hline \multicolumn{4}{|l|}{ Race } \\
\hline White & Reference & & \\
\hline Black & 0.69 & $0.31-1.56$ & 0.377 \\
\hline Others & 0.40 & $0.24-0.70$ & 0.001 \\
\hline Unknown & 0.12 & $0.02-0.87$ & 0.036 \\
\hline \multicolumn{4}{|l|}{ Tumor size(cm) } \\
\hline$\leq 2$ & Reference & & \\
\hline$>2$ & 1.53 & $1.11-2.10$ & 0.009 \\
\hline Unknown & 1.27 & $1.00-1.62$ & 0.055 \\
\hline \multicolumn{4}{|l|}{ Primary site } \\
\hline Labium majus & Reference & & \\
\hline Labium minus & 1.66 & $0.80-3.47$ & 0.176 \\
\hline Clitoris & 1.11 & $0.35-3.50$ & 0.865 \\
\hline Overlapping lesion of vulva & 1.88 & $0.76-4.65$ & 0.171 \\
\hline Vulva NOS & 0.88 & $0.71-1.11$ & 0.282 \\
\hline SEER Historic Stage A & & & 0.112 \\
\hline Localized & Reference & & \\
\hline Regional & 1.12 & $0.64-1.97$ & 0.685 \\
\hline Distant & 10.24 & $3.22-32.59$ & 0.000 \\
\hline Unknown & 1.27 & $0.94-1.71$ & 0.117 \\
\hline
\end{tabular}

BCC were females, and the female to male ratio was 5.3. The reason for this female predominance remains to be clarified. One possible explanation is the presence of a chronic skin irritation such as chronic vulvovaginitis, since most patients with vulvar BCC tend to be postmenopausal with drastically reduced hormone levels (16). Despite the higher incidence,
TABLE 4 | Multivariate analyses of overall survival for male genital basal cell carcinoma.

\begin{tabular}{lccc}
\hline Characteristics & HR & $\mathbf{9 5 \%} \mathbf{C l}$ & P-value \\
\hline Age & & & \\
$\leq 60$ & Reference & & \\
$61-70$ & 3.25 & $1.15-9.18$ & 0.026 \\
$71-80$ & 5.51 & $2.03-14.94$ & 0.001 \\
$>80$ & 16.58 & $6.24-44.04$ & 0.000 \\
Race & & & \\
White & Reference & & \\
Black & 0.38 & $0.05-2.90$ & 0.351 \\
Others & 0.75 & $0.18-3.17$ & 0.697 \\
Unknown & 0.40 & $0.05-2.90$ & 0.362 \\
Tumor size(cm) & & & \\
$\quad \leq 2$ & Reference & & \\
$>2$ & 1.38 & $0.57-3.34$ & 0.478 \\
Unknown & 0.70 & $0.39-1.26$ & 0.230 \\
Primary site & & & \\
Scrotum & Reference & & \\
Penis & 1.76 & $1.06-2.92$ & \\
SEER Historic Stage A & & & \\
Localized & Reference & & \\
Regional & 3.77 & $1.12-12.71$ & 0.032 \\
Distant & 62.04 & $11.10-346.88$ & 0.000 \\
Unknown & 0.64 & $0.26-1.63$ & 0.353 \\
\hline
\end{tabular}

females had better DSS than males, and the cause remains to be elucidated.

Most BCCs are indolent, only infiltrate locally, and rarely metastasize (18). As a result of delayed visits and diagnosis, the tumor size of genital BCCs tends to be large. Patients may hesitate to seek treatment because of the privacy of genital area. In addition, the presentation of vulvar BCC is variable and nonspecific, such as itching, a lump, bleeding, and pain $(19,20)$. 
Physicians may initially mistake lesions for inflammatory or infectious dermatoses (21). In this study, there were 718 cases with known size (mean $1.77 \mathrm{~cm}$ ), and tumors greater than $2 \mathrm{~cm}$ accounted for $27.2 \%$. For women, tumors over $2 \mathrm{~cm}$ conferred worse prognoses (HR 1.53 [95\% CI 1.11-2.10]) than those less than $2 \mathrm{~cm}$ on multivariate analysis. Only four cases in this cohort had distant metastasis; all of them died of diseasespecific death with survival ranging from 0 to 27 months (average 9.8 months). Thus, although rare, genital BCC still has the risk of distant metastasis, and the prognosis is very poor once it occurs. Additionally, the skin lesions on the genital area should be differentiated from malignancy such as squamous cell carcinoma, malignant melanoma, Bowen's disease, and extramammary Paget (22). It is recommended that all suspect lesions on the genital skin be biopsied as soon as possible to confirm the diagnosis and improve the prognosis.

The major histologic presentation of genital BCCs in both men and women was nodular subtype, which was similar to non-genital BCC (23). In this study, there was no statistical difference in survival between histologic subtypes by Kaplan-Meier survival analysis and multivariate analysis. According to the World Health Organization's coding and classification of BCC, different histologic subtypes share the same code (such as $8097 / 3$ for micronodular and nodular subtype), which may affect the results of survival analysis to some extent. Additionally, we did not include basal cell adenocarcinoma in our study due to its low incidence in the genital area and the small number of cases in the SEER database. Mitchell has conducted a population-based survival analysis on basal cell adenocarcinoma, while patients with the involvement of penis, scrotum, and vulva accounted for only $0.4,0.8$, and $10.1 \%$, respectively. Therefore, it is difficult to obtain survival data of genital BCC from this research.

Consistent with previous reports (24), in this cohort, most vulvar BCCs occurred on the labia majus, accounting for $89.6 \%$ of all vulvar BCC cases with known explicit location. BCCs of the penis were less frequently reported than BCCs of the scrotum (25), and in this cohort, the total number of penis BCCs accounted for only one-third of the scrotum BCCs. We found that penis BCCs showed poorer prognosis than scrotum BCC, with about 1.8 times higher risk of death. In addition, only two male patients with distant disease were identified, and all occurred on the penis. To our knowledge, this is the first comparison conducted on genital BCCs between penis and scrotum, and further research is needed to verify this conclusion.

Our study does have some limitations. Several known factors such as margin of resection, surgical modalities, depth of invasion, genetic factors, comorbid conditions, systemic treatment, and the time interval from onset of symptoms to diagnosis are not available in the SEER database. These variables are generally considered predictors of prognosis. BCC is an indolent skin malignancy with high recurrence but few metastases. Given that the SEER database does not contain information regarding disease recurrence, we cannot determine recurrence rates and recurrence-free survival. In addition, we were unable to calculate the metastasis rates and identify metastasis-related risk factors due to the missing and obscure information on metastasis. Therefore, it is difficult to conduct in-depth research to determine whether the biological behavior of genital BCCs is more aggressive than BCCs located on extragenital skin.

As mentioned previously, different histologic subtypes share the same code, causing misclassification in this study. Furthermore, the primary site coding was ambiguous (i.e., "Overlapping lesion of vulva" and "Vulva NOS"), and data on tumor size and stage are largely missing, so these patients could not be included in specific analysis by site, size, and stage, respectively. In addition, the SEER database does not specify the age of patients over 85 years, and we replaced it with 85 in calculation, which may lead to an underestimation of the average age. It is important to emphasize that our research was based on US population data, and our results may not be universally applicable to the world. Finally, these data are retrospective and prospective research is still necessary to further verify the results of this study.

Despite the above limitations, this population-based study represents the first large-scale analysis of genital BCC, providing more insight and comprehensive understanding into this rare skin cancer, and encouraging follow-up research.

\section{CONCLUSION}

This study offers a comprehensive analysis on genital BCC from a well-defined population. In general, genital BCCs have great prognosis, but the survival becomes very poor once distant metastasis occurs. Despite similar clinicopathologic features and OS outcomes, men and women should be treated as two different entities when making survival predictions.

\section{DATA AVAILABILITY STATEMENT}

The datasets presented in this study can be found in online repositories. The names of the repository/repositories and accession number(s) can be found below: (http://www.seer. cancer.gov).

\section{ETHICS STATEMENT}

Ethical review and approval was not required for the study on human participants in accordance with the local legislation and institutional requirements. Written informed consent for participation was not required for this study in accordance with the national legislation and the institutional requirements.

\section{AUTHOR CONTRIBUTIONS}

$\mathrm{XC}$ drafted the manuscript. YH participated in the design of the manuscript and made relevant statistical analysis. CC collected the related literature. All authors contributed to the article and approved the submitted version. 


\section{REFERENCES}

1. Rogers HW, Weinstock MA, Harris AR, Hinckley MR, Feldman SR, Fleischer AB, et al. Incidence estimate of nonmelanoma skin cancer in the United States, 2006. Arch Dermatol (2010) 146(3):283-7. doi: 10.1001/archdermatol.2010.19

2. Mulayim N, Foster Silver D, Tolgay Ocal I, Babalola E. Vulvar basal cell carcinoma: two unusual presentations and review of the literature. Gynecol Oncol (2002) 85(3):532-7. doi: 10.1006/gyno.2001.6582

3. Rubin AI, Chen EH, Ratner D. Basal-cell carcinoma. N Engl J Med (2005) 353 (21):2262-9. doi: 10.1056/NEJMra044151

4. Dai B, Kong YY, Ye DW, Xu XW, Yao XD, Zhang SL. Basal cell carcinoma of the scrotum: clinicopathologic analysis of 10 cases. Dermatol Surg (2012) 38 (5):783-90. doi: 10.1111/j.1524-4725.2012.02356.x

5. Loh TY, Rubin AG, Jiang SI. Basal Cell Carcinoma of the Dorsal Foot: An Update and Comprehensive Review of the Literature. Dermatol Surg (2017) 43(1):32-9. doi: 10.1097/DSS.0000000000000900

6. Verkouteren JAC, Ramdas KHR, Wakkee M, Nijsten T. Epidemiology of basal cell carcinoma: scholarly review. Br J Dermatol (2017) 177(2):359-72. doi: 10.1111/bjd.15321

7. Moriwaki S, Ray S, Tarone RE, Kraemer KH, Grossman L. The effect of donor age on the processing of UV-damaged DNA by cultured human cells: reduced DNA repair capacity and increased DNA mutability. Mutat Res (1996) 364 (2):117-23. doi: 10.1016/0921-8777(96)00029-8

8. Nahass GT, Blauvelt A, Leonardi CL, Penneys NS. Basal cell carcinoma of the scrotum. Report of three cases and review of the literature. J Am Acad Dermatol (1992) 26(4):574-8. doi: 10.1016/0190-9622(92)70083-r

9. Kwasniak LA, Garcia-Zuazaga J. Basal cell carcinoma: evidence-based medicine and review of treatment modalities. Int J Dermatol (2011) 50 (6):645-58. doi: 10.1111/j.1365-4632.2010.04826.x

10. Kandamany N, Monk B. Basal cell carcinoma presenting late in a shotgun scar. Am J Clin Dermatol (2009) 10(4):271-2. doi: 10.2165/00128071200910040-00009

11. Li C, Athar M. Ionizing Radiation Exposure and Basal Cell Carcinoma Pathogenesis. Radiat Res (2016) 185(3):217-28. doi: 10.1667/RR4284.S1

12. Gibson GE, Ahmed I. Perianal and genital basal cell carcinoma: A clinicopathologic review of 51 cases. J Am Acad Dermatol (2001) 45(1):6871. doi: $10.1067 / \mathrm{mjd} .2001 .114588$

13. Iannacone MR, Gheit T, Waterboer T, Giuliano AR, Messina Jl, Fenske NA, et al. Case-control study of cutaneous human papillomavirus infection in Basal cell carcinoma of the skin. J Invest Dermatol (2013) 133(6):1512-20. doi: 10.1038/ jid.2012.478

14. Leonardi G, Vahter M, Clemens F, Goessler W, Gurzau E, Hemminki K, et al. Inorganic arsenic and basal cell carcinoma in areas of Hungary, Romania, and
Slovakia: a case-control study. Environ Health Perspect (2012) 120(5):721-6. doi: 10.1289/ehp.1103534

15. Surdu S. Non-melanoma skin cancer: occupational risk from UV light and arsenic exposure. Rev Environ Health (2014) 29(3):255-64. doi: 10.1515/ reveh-2014-0040

16. Takahashi H. Non-ulcerative basal cell carcinoma arising on the genitalia. J Dermatol (2000) 27(12):798-801. doi: 10.1111/j.1346-8138.2000.tb02285.x

17. Brooke RC. Basal cell carcinoma. Clin Med (Lond) (2005) 5(6):551-4. doi: 10.7861/clinmedicine.5-6-551

18. Namuduri RP, Lim TY, Yam PK, Gatsinga R, Lim-Tan SK, Chew SH, et al. Vulvar basal cell carcinoma: clinical features and treatment outcomes from a tertiary care centre. Singapore Med J (2019) 60(9):479-82. doi: 10.11622/ smedj. 2019014

19. Fleury AC, Junkins-Hopkins JM, Diaz-Montes T. Vulvar basal cell carcinoma in a 20-year-old: Case report and review of the literature. Gynecol Oncol Case Rep (2011) 2(1):26-7. doi: 10.1016/j.gynor.2011.11.005

20. Ghurani GB, Penalver MA. An update on vulvar cancer. Am J Obstet Gynecol (2001) 185(2):294-9. doi: 10.1067/mob.2001.117401

21. De Giorgi V, Massi D, Lotti T. Basal-cell carcinoma. N Engl J Med (2006) 354 (7):769-71. doi: 10.1056/NEJMc053488

22. Verhoeven RH, Aben KK, van Rossum MM, Reedijk AM, Botterweck AA, Veerbeek L, et al. New insights into the aetiology of scrotal cancer, a nationwide case-control study in the Netherlands. J Eur Acad Dermatol Venereol (2014) 28(1):65-71. doi: 10.1111/jdv.12056

23. Kim DP, Kus KJB, Ruiz E. Basal Cell Carcinoma Review. Hematol Oncol Clin North Am (2019) 33(1):13-24. doi: 10.1016/j.hoc.2018.09.004

24. Saito S, Hata H, Inamura Y, Kitamura S, Yanagi T, Shimizu H. Vulvar basal cell carcinoma with adhesion of the labia majora and minora. Clin Exp Dermatol (2017) 42(1):92-3. doi: 10.1111/ced.12956

25. Roewe RJ, Uhlman MA, Bockholt NA, Gupta A. Basal cell carcinoma of the penis: a case report and review of the literature. Case Rep Urol (2014) 2014:173076. doi: $10.1155 / 2014 / 173076$

Conflict of Interest: The authors declare that the research was conducted in the absence of any commercial or financial relationships that could be construed as a potential conflict of interest.

Copyright (c) 2021 Chen, Hou, Chen and Jiang. This is an open-access article distributed under the terms of the Creative Commons Attribution License (CC BY). The use, distribution or reproduction in other forums is permitted, provided the original author(s) and the copyright owner(s) are credited and that the original publication in this journal is cited, in accordance with accepted academic practice. No use, distribution or reproduction is permitted which does not comply with these terms. 